\title{
Computation of quantum-transport properties
}

by random-matrix theory

\author{
C. W. J. Beenakker \\ Instituut-Lorentz \\ University of Leiden \\ P.O. Box 9506 \\ NL-2300 RA Leiden
}

The Netherlands

ABSTRACT. A random-matrix formula is derived for the variance of an arbitrary linear statistic on the transmission eigenvalues. The variance is independent of the eigenvalue density and has a universal dependence on the symmetry of the matrix ensemble. The formula generalizes the Dyson-Mehta theorem in the statistical theory of energy levels. It demonstrates that the universality of the conductance fluctuations is generic for a whole rlass of transport properties in mesoscopic systems.

\section{Introduction}

The theory of universal conductance fluctuations in disordered metals was originally formulated as a diagrammatic perturbation theory in terms of Greens functions $[1,2]$. Subsequently, an alternative non-perturbative theory was developed, based on the properties of random scattering (or transfer) matrices $[3,4,5,6]$. The universality of the sample-to-sample fluctuations in the conductance was shown to be a manifestation of the universal eigenvalue repulsion in random-matrix ensembles, discovered long ago in nuclear physics $[7,8]$. The symmetry class of the ensemble manifests itself as a universal dependence of the variance of the conductance on the presence of time-reversal symmetry and/or spin-orbit scattering.

Computationally, the random-matrix theory of quantum transport is not as well developed as its counterpart in nuclear physics. In that field there is a celebrated formula, due to Dyson and Mehta [9], which allows one to calculate analytically the variance of any linear statistic on the energy levels (i.e. an observable $A=\sum_{n} a\left(E_{n}\right)$, with $a$ an arbitrary function of energy). The Dyson-Mehta formula reads 


$$
\operatorname{Var} A=\frac{1}{\beta} \frac{1}{\pi^{2}} \int_{0}^{\infty} d k|a(k)|^{2} k,
$$

where $a(k)=\int_{-\infty}^{\infty} d E \mathrm{e}^{\mathrm{i} k E} a(E)$ is the Fourier transform of $a(E)$. The parameter $\beta$ characterizes the symmetry class of the random-matrix ensemble (see below). No such formula exists for universal fluctuations in transport properties. The crucial difference, which impedes any obvious generalization of the Dyson-Mehta formula, is that the correlation function of transmission eigenvalues is not translationally invariant [6]. The lack of a simple analytical technique in the random-matrix theory of quantum transport is being felt especially now that universal fluctuations in transport properties other than the conductance (both in conductors and in superconductors) have become of interest. Examples are the critical-current fluctuations in Josephson junctions $[10]$, conductance fluctuations at normal-superconductor interfaces [11], and fluctuations in the shot-noise power of metals [12].

Recently we have been able to overcome this obstacle to a generalization of the Dyson-Mehta formula [13]. In the present contribution we discuss our method and our main results. A more comprehensive presentation will be published elsewhere [14].

\section{Formulation of the problem}

We consider a disordered conductor of length $L$ and width $W$ at zero temperature. The elastic scattering of an electron at the Fermi level is described by the unitary scattering matrix ( $s$-matrix)

$$
s=\left(\begin{array}{cc}
r_{11} & t_{12} \\
t_{21} & r_{22}
\end{array}\right)
$$

The reflection and transmission matrices $r$ and $t$ are $N \times N$ matrices, $N$ being the number of propagating modes at the Fermi energy. The matrix product $t_{12} t_{12}^{\dagger}$ is hermitean, and hence has real eigenvalues $T_{n}(n=1,2, \ldots N)$. Since $t_{12} t_{12}^{\dagger}=r_{11} t_{21}^{\dagger} t_{21} r_{11}^{-1}$ (as follows from unitarity of $s$ ), the matrices $t_{12} t_{12}^{\dagger}$ and $t_{21} t_{21}^{\dagger}$ have the same set of eigenvalues. We refer to the $T_{n}$ 's as the transmission eigenvalues. Unitarity of $s$ also implies that $0 \leq T_{n} \leq 1$ for all $n$. We will study transport properties $A$ of the form

$$
A=\sum_{n=1}^{N} f\left(T_{n}\right) .
$$

A quantity of the form (3) is called a linear statistic on the transmission eigenvalues. The word "linear" indicates that $A$ does not contain products of different eigenvalues, but the function $f(T)$ may well depend non-linearly on $T$.
Starting point of our analysis is the joint probability distribution of transmission eigenvalues obtained in the random-matrix theory of quantum transport $[5,6]$. To make contact with that theory we adopt the parametrization

$$
T_{n} \equiv \frac{1}{1+\lambda_{n}}, 0 \leq \lambda<\infty,
$$

and work with a linear statistic on the $\lambda$ 's

$$
A=\sum_{n=1}^{N} a\left(\lambda_{n}\right) .
$$

Since there is a simple one-to-one relationship between $\lambda$ and $T$, we will still refer to the $\lambda$ 's as "transmission eigenvalues". The distribution of the $\lambda$ 's is given by [5]

$$
\begin{aligned}
& P\left(\left\{\lambda_{n}\right\}\right)=Z^{-1} \exp \left[-\beta \mathcal{H}\left(\left\{\lambda_{n}\right\}\right)\right], \\
& \mathcal{H}\left(\left\{\lambda_{n}\right\}\right)=-\sum_{i<j} \ln \left|\lambda_{i}-\lambda_{j}\right|+\sum_{i} V\left(\lambda_{i}\right),
\end{aligned}
$$

where $Z$ is such that $P$ is normalized to unity,

$$
Z=\int_{0}^{\infty} d \lambda_{1} \cdots \int_{0}^{\infty} d \lambda_{N} \exp \left[-\beta \mathcal{H}\left(\left\{\lambda_{n}\right\}\right)\right]
$$

The parameter $\beta$ depends on the symmetry properties of the ensemble of scattering matrices. If time-reversal symmetry is broken (by a magnetic field), $\beta=2$. In the presence of time-reversal symmetry, $\beta=1$ if the scattering is spin-independent, while $\beta=4$ for strong spin-orbit scattering. These three universality classes for the scattering matrix correspond in the statistical theory of energy levels to the orthogonal $(\beta=1)$, unitary $(\beta=2)$, and symplectic $(\beta=4)$ ensembles [7].

The probability distribution (6) is based on (a) an isotropy assumption, which implies that flux incident in one scattering channel is, on average, equally distributed among all outgoing channels; and (b) a maximum entropy hypothesis, which yields (6) as the least restrictive distribution consistent with a given mean eigenvalue density. The potential $V$ in Eq. (6) then plays the role of a Lagrange multiplier, which has to be chosen in such a way that $P$ yields the required eigenvalue density. Assumption (a) requires a conductor much longer than wide, i.e. the quasi-one-dimensional limit $L \gg$ $W$. Assumption (b) has been justified by numerical simulations $[5,6]$, but there is no rigorous proof of its validity. As we will discuss, one of the implications of our results will be that Eq. (6) is not rigorously valid although the error is quite small.

The goal of our analysis is to obtain the variance $\operatorname{Var} A$ of the linear statistic (5) from the eigenvalue distribution function (6). To this end we need 
to know how pairs of transmission eigenvalues are correlated. These correlations are strong because of the logarithmic repulsion of the eigenvalues in the "hamiltonian" (6). The logarithmic interaction has a fundamental geometric origin: It is the jacobian associated with the transformation from the space of scattering matrices to the smaller space of transmission eigenvalues $[4,5,6]$. Our approach is to relate the correlation function to a functional derivative of the mean eigenvalue density with respect to $V$, and then to evaluate this functional derivative in the limit $N \rightarrow \infty$. In this section wc deal with the first step of this program, which is an exercise in statistical mechanics. A similar line of reasoning was used by Politzer [15], to show that $A$ has a gaussian distribution in the limit $N \rightarrow \infty$.

The mean density of transmission eigenvalues $\langle\rho(\lambda)\rangle$ is defined as the ensemble average of the microscopic density:

$$
\begin{aligned}
\rho(\lambda) & =\sum_{n=1}^{N} \delta\left(\lambda-\lambda_{n}\right), \\
\langle\rho(\lambda)\rangle & =\frac{\int d \lambda_{1} \cdots \int d \lambda_{N} \rho(\lambda) \exp (-\beta \mathcal{H})}{\int d \lambda_{1} \cdots \int d \lambda_{N} \exp (-\beta \mathcal{H})} .
\end{aligned}
$$

We define the "two-point correlation function" $K_{2}\left(\lambda, \lambda^{\prime}\right)$ by

$$
K_{2}\left(\lambda, \lambda^{\prime}\right)=\langle\rho(\lambda)\rangle\left\langle\rho\left(\lambda^{\prime}\right)\right\rangle-\left\langle\rho(\lambda) \rho\left(\lambda^{\prime}\right)\right\rangle .
$$

It is related to the "two-level cluster function" $T_{2}\left(\lambda, \lambda^{\prime}\right)$ of Ref. [7] by

$$
K_{2}\left(\lambda, \lambda^{\prime}\right)=T_{2}\left(\lambda, \lambda^{\prime}\right)-\langle\rho(\lambda)\rangle \delta\left(\lambda-\lambda^{\prime}\right) .
$$

We include the singular self-correlation in the correlation function because it contributes to the variance of a linear statistic (see below).

To obtain the required relationship, we take the functional derivative of $\langle\rho(\lambda)\rangle$ with respect to $V\left(\lambda^{\prime}\right)$. Since $\delta \mathcal{H} / \delta V(\lambda)=\rho(\lambda)$, differentiation of Eq. (9) yields

$$
\begin{aligned}
\frac{\delta\langle\rho(\lambda)\rangle}{\delta V\left(\lambda^{\prime}\right)}= & -\beta \frac{\int d \lambda_{1} \cdots \int d \lambda_{N} \rho(\lambda) \rho\left(\lambda^{\prime}\right) \exp (-\beta \mathcal{H})}{\int d \lambda_{1} \cdots \int d \lambda_{N} \exp (-\beta \mathcal{H})} \\
+ & \beta\left(\frac{\int d \lambda_{1} \cdots \int d \lambda_{N} \rho(\lambda) \exp (-\beta \mathcal{H})}{\int d \lambda_{1} \cdots \int d \lambda_{N} \exp (-\beta \mathcal{H})}\right) \\
& \times\left(\frac{\int d \lambda_{1} \cdots \int d \lambda_{N} \rho\left(\lambda^{\prime}\right) \exp (-\beta \mathcal{H})}{\int d \lambda_{1} \cdots \int d \lambda_{N} \exp (-\beta \mathcal{H})}\right) \\
= & -\beta\left\langle\rho(\lambda) \rho\left(\lambda^{\prime}\right)\right\rangle+\beta\langle\rho(\lambda)\rangle\left\langle\rho\left(\lambda^{\prime}\right)\right\rangle .
\end{aligned}
$$

Hence we obtain the key relation

$$
K_{2}\left(\lambda, \lambda^{\prime}\right)=\frac{1}{\beta} \frac{\delta\langle\rho(\lambda)\rangle}{\delta V\left(\lambda^{\prime}\right)} .
$$

The linear statistic (5) can be written in terms of the microscopic eigenvalue density (8),

$$
A=\int_{0}^{\infty} d \lambda a(\lambda) \rho(\lambda)
$$

The ensemble average $\langle A\rangle$ is

$$
\langle A\rangle=\int_{0}^{\infty} d \lambda a(\lambda)\langle\rho(\lambda)\rangle,
$$

so that the variance $\operatorname{Var} A \equiv\left\langle A^{2}\right\rangle-\langle A\rangle^{2}$ becomes

$$
\begin{aligned}
\operatorname{Var} A & =-\int_{0}^{\infty} d \lambda \int_{0}^{\infty} d \lambda^{\prime} a(\lambda) a\left(\lambda^{\prime}\right) K_{2}\left(\lambda, \lambda^{\prime}\right) \\
& =-\frac{1}{\beta} \int_{0}^{\infty} d \lambda \int_{0}^{\infty} d \lambda^{\prime} a(\lambda) a\left(\lambda^{\prime}\right) \frac{\delta\langle\rho(\lambda)\rangle}{\delta V\left(\lambda^{\prime}\right)} .
\end{aligned}
$$

This relationship between the variance of a linear statistic and the functional derivative of the density of transmission eigenvalues is an exact consequence of the probability distribution (6).

$\Lambda \mathrm{n}$ immediate implication of Eq. (16) is that $\operatorname{Var} A \propto 1 / \beta$ for all linear statistics, if and only if the functional derivative $\delta\langle\rho\rangle / \delta \mathrm{V}$ is independent of the symmetry parameter $\beta$. As we will see in the next section, this is indeed the case for $N \rightarrow \infty$. Furthermore, since all microscopic details of the system snter via the "potential" $V(\lambda)$, universality of the fluctuations is obtained if $\langle\rho\rangle$ is a lincar functional of $V$. Again, this holds for $N \rightarrow \infty$, as we will sne next.

\section{Integral equation for the eigenvalue density}

To cvaluate the functional derivative (13) we must know how the density of transmission eigenvalues $\langle\rho\rangle$ depends on the potential $V$ in the hamiltonian (6). This problem has been addressed before in the random-matrix theory of energy levels, which is also based on the distribution function (6), but without the positivity constraint on $\lambda$. For that case, Dyson [16] has derived the following equation,

$$
\int d \lambda^{\prime}\left\langle\rho\left(\lambda^{\prime}\right)\right\rangle \ln \left|\lambda-\lambda^{\prime}\right|+\frac{\beta-2}{2 \beta} \ln \langle\rho(\lambda)\rangle=V(\lambda)+\text { const },
$$

where the additive constant is to be determined from the normalization condition

$$
\int d \lambda\langle\rho(\lambda)\rangle=N
$$


The second term on the 1.h.s. of Eq. (17) is of order $N^{-1} \ln N$ relative to the first, and terms of still higher order in $1 / N$ are neglected. To calculate the two-point correlation function (13) in leading order it is sufficient to retain only the first term, so that we can work with the linear integral equation

$$
\int d \lambda^{\prime}\left\langle\rho\left(\lambda^{\prime}\right)\right\rangle \ln \left|\lambda-\lambda^{\prime}\right|=V(\lambda)+\text { const. }
$$

Eq. (19) has the intuitive "mean-field" interpretation (originally due to Wigner), that the "charge density" $\langle\rho\rangle$ adjusts itself to the "external potential" $V$ in such a way that the total force on any charge $\lambda$ vanishes [6]. The more accurate equation (17) shows that, in fact, Eq. (19) is the leading term in a $1 / N$ expansion.

In Dyson's derivation of Eq. (17), essential use is made of the fact that all integrals run from $-\infty$ to $+\infty$. In our case, the integration range is from 0 to $\infty$. We have verified that the positivity constraint on $\lambda$ does not introduce any extra terms in the integral equation, to the order considered [14].

To obtain the two-point correlation function $K_{2}\left(\lambda, \lambda^{\prime}\right)$ in the limit $N \rightarrow$ $\infty$ we thus need to consider the integral equation (19). The functional derivative $\delta\langle\rho\rangle / \delta V$ equals the solving kernel of

$$
\int_{0}^{\infty} d \lambda^{\prime} \psi\left(\lambda^{\prime}\right) \ln \left|\lambda-\lambda^{\prime}\right|=\phi(\lambda)+\text { const }
$$

where the additive constant has to be chosen such that $\psi$ has zero mean,

$$
\int_{0}^{\infty} d \lambda \psi(\lambda)=0
$$

since the variations in $\langle\rho\rangle$ have to occur at constant $N$. Because of Eq. (13), the integral solution

$$
\psi(\lambda)=\int_{0}^{\infty} d \lambda^{\prime} \beta K_{2}\left(\lambda, \lambda^{\prime}\right) \phi\left(\lambda^{\prime}\right)
$$

of Eq. (20) directly determines the two-point correlation function, and hence the variance (16) of any linear statistic. Since the integral equation (20) does not contain any microscopic parameters, and is independent of the symmetry parameter $\beta$, the two statements of universality made at the end of the previous section are now validated: $\operatorname{Var} A$ depends on $\beta$ as $1 / \beta$ and is independent of microscopic parameters.

\section{Formula for the variance of a linear statistic}

To calculate the value of $\operatorname{Var} A$ we have to determine the solving kernel of Eq. (20). This can be done by a Mellin transformation. Here we give only the result, mathematical details of the calculation will be published elsewher
[14]. The two-point correlation function $K_{2}\left(\lambda, \lambda^{\prime}\right)$ which follows from Eqs. (20) and (22) is

$$
K_{2}\left(\lambda, \lambda^{\prime}\right)=\frac{1}{\pi^{2} \beta} \frac{\partial}{\partial \lambda} \frac{\partial}{\partial \lambda^{\prime}} \ln \left|\frac{\sqrt{ } \lambda-\sqrt{ } \lambda^{\prime}}{\sqrt{ } \lambda+\sqrt{ } \lambda^{\prime}}\right| .
$$

This function is obviously not translationally invariant. However, a translationally invariant kernel can be obtained by the transformation $\lambda=\mathrm{e}^{x}$, $\tilde{K}_{2}\left(x, x^{\prime}\right)=\mathrm{e}^{x+x^{\prime}} K_{2}\left(\mathrm{e}^{x}, \mathrm{e}^{x^{\prime}}\right)$. The result is

$$
\tilde{K}_{2}\left(x, x^{\prime}\right)=-\frac{1}{\pi^{2} \beta} \frac{d^{2}}{d\left(x-x^{\prime}\right)^{2}} \ln \left|\tanh \frac{x-x^{\prime}}{4}\right| .
$$

Substituting Eq. (23) into Eq. (16), and carrying out two partial integrations, we find the formula

$$
\operatorname{Var} A=-\frac{1}{\beta} \frac{1}{\pi^{2}} \int_{0}^{\infty} d \lambda \int_{0}^{\infty} d \lambda^{\prime} \ln \left|\frac{\sqrt{ } \lambda-\sqrt{ } \lambda^{\prime}}{\sqrt{ } \lambda+\sqrt{ } \lambda^{\prime}}\right| \frac{d a(\lambda)}{d \lambda} \frac{d a\left(\lambda^{\prime}\right)}{d \lambda^{\prime}}
$$

In an equivalent Fourier representation, we can write

$$
\operatorname{Var} A=\frac{1}{\beta} \frac{1}{\pi^{2}} \int_{0}^{\infty} d k|\tilde{a}(k)|^{2} k \tanh (\pi k)
$$

Here $\tilde{a}(k)$ is the Mellin transform of $a(\lambda)$, i.e. the Fourier transform with respect to $x \equiv \ln \lambda$ :

$$
\tilde{a}(k)=\int_{0}^{\infty} d \lambda \lambda^{\mathrm{i} k-1} a(\lambda)=\int_{-\infty}^{\infty} d x \mathrm{e}^{\mathrm{i} k x} a\left(\mathrm{e}^{x}\right) .
$$

The kernel in Eq. (26) is the Fourier transform with respect to $x-x^{\prime}$ of $\tilde{K}_{2}\left(x, x^{\prime}\right)$. Eq. (26) is for the quantum transport problem what the DysonMehta formula (1) was for the problem of the statistics of energy levels.

As an independent check of the validity of our key result, we have compared Eq. (26) with an exactly solvable model. This is the Laguerre ensemble, defined by Eq. (6) with $\beta=2$ and $V(\lambda)=\frac{1}{2} \lambda-\frac{1}{2} \alpha \ln \lambda$. The parameter $\alpha>-1$ is arbitrary. The correlation function for this ensemble is known exactly, in terms of generalized Laguerre polynomials $[6,17]$. The variance can then be obtained by doing the double integration in Eq. (16) numerically. In Fig. 1 we show the comparison for the variance of the conductance. The conductance $G$ is a linear statistic on the transmission eigenvalues, according to the Landaver formula

$$
\left(i / C_{0}=\sum_{n=1}^{N} T_{n}=\sum_{n=1}^{N} \frac{1}{1+\lambda_{n}} .\right.
$$

Here $G_{0}=2 e^{2} / h$ is the conductance quantum. The Mellin transform of $a(\lambda)=(1+\lambda)^{-1}$ is 


\subsection{JOSEPHSON JUNCTION}

The supercurrent-phase relationship $I(\phi)$ of a point-contact Josephson junction can be expressed as well in terms of the normal-state transmission eigenvalues [10],

$$
I(\phi)=\frac{e \Delta}{2 \hbar} \sum_{n=1}^{N} \frac{T_{n} \sin \phi}{\left[1-T_{n} \sin ^{2}(\phi / 2)\right]^{1 / 2}},
$$

$\Delta$ being the energy gap in the bulk superconductor. In this case it is more convenient to evaluate the variance formula (25), rather than Eq. (26). The quantity of most direct experimental significance is the critical current $I_{\mathrm{c}} \equiv$ $\max I(\phi)$. In Ref. [21] it is shown that, for $N \rightarrow \infty, \operatorname{Var} I_{\mathrm{c}}=\operatorname{Var} I\left(\phi_{\mathrm{c}}\right)$, where $\phi_{c}=1.97$ is the phase difference at which the ensemble average $\langle I(\phi)\rangle$ reaches its maximum. By numerically integrating Eq. (25) we find $\operatorname{Var} I_{\mathrm{c}}=$ $0.085(e \Delta / \hbar)^{2}$.

\section{Summary}

In conclusion, we have derived the analogue of the Dyson-Mehta theorem [9] for the quantum transport problem. The formula obtained [Eq. (25) or (26)] demonstrates that the universality which was the hallmark of the phenomenon of "universal conductance fluctuations" $[1,2]$ is generic for linear statistics on the transmission eigenvalues. This universality was anticipated [3] from the random-matrix theory of energy levels, but could not previously be established because of the absence of translational invariance of the correlation function of transmission coefficients (originating from the unitarity of the scattering matrix) [6]. Finally, our analysis has revealed a small but real numerical discrepancy between the random-matrix theory [5] and the diagrammatic calculation [2], which implies that the interaction between the $\lambda$ eigenvalues is not precisely logarithmic.

\section{Acknowledgements}

I have benefitted from discussions with R. A. Jalabert, M. J. M de Jong, J. M. J. van Leeuwen, B. Rejaei, and A. D. Stone. I thank R. A. Jalabcrt for helping me with the numerical integrations. This research was supported financially by the "Nederlandse organisatie voor Wetenschappelijk Onderzoek" (NWO) via the "Stichting voor Fundamenteel Onderzoek der Materio" (FOM).

\section{References}

[1] B. L. Al'tshuler, Pis'ma Zh. Eksp. Teor. Fiz. 41, 530 (1985) [JETP Lett. 41, 648, (1985)].

[2] P. A. Lee and A. D. Stone, Phys. Rev. Lett. 55, 1622 (1985).

[3] Y. Imry, Europhys. Lett. 1, 249 (1986).

[1] P. A. Mello, P. Pereyra, and N. Kumar, Ann. Phys. 181, 290 (1988).

[5] K. A. Muttalib, J.-L. Pichard, and A. D. Stone, Phys. Rev. Lett. 59, 2475 (1987).

[6] A. D. Stone, P. A. Mello, K. A. Muttalib, and J.-L. Pichard, in: Mesoscopic Phenomena in Solids, ed. by B. L. Al'tshuler, P. A. Lee, and R. A. Webb (North-Holland, Amsterdam, 1991).

[7] M. L. Mehta, Random Matrices and the Statistical Theory of Energy Levels (Academic, New York, 1967).

[8] T. A. Brody, J. Flores, J. B. French, P. A. Mello, A. Pandey, S. S. M. Wong, Rev. Mod. Phys. 53, 385 (1981).

[9] F. J. Dyson and M. L. Mehta, J. Math. Phys. 4, 701 (1963).

[10] C. W. J. Beenakker, Phys. Rev. Lett. 67, 3836 (1991); 68, 1442(E) (1992).

[11] Y. Takane and H. Ebisawa, J. Phys. Soc. Japan 60, 3130 (1991); 61, 2858 (1992).

[12] M. J. M. de Jong and C. W. J. Beenakker, Phys. Rev. B (November 15, 1992).

[13] C. W. J. Beenakker, submitted for publication to Phys. Rev. Lett.

[14] C. W. J. Beenakker, to be published.

[15] H. D. Politzer, Phys. Rev. B 40, 11917 (1989).

[16] F. J. Dyson, J. Math. Phys. 13, 90 (1972).

[17] K. Slevin, J.-L. Pichard, and P. A. Mello, Europhys. Lett. 16, 649 (1991).

[18] P. A. Mello, Phys. Rev. Lett. 60, 1089 (1988); P. A. Mello and A. D. Stone, Phys. Rev. B 44, 3559 (1991).

[19] M. Büttiker, Phys. Rev. Lett. 65, 2901 (1990).

[20] C. W. J. Beenakker, Phys. Rev. B (December 15, 1992).

[21] C. W. J. Beenakker, in: Transport Phenomena in Mesoscopic Systems, Springer Series in Solid State Sciences, Vol. 109, ed. by H. Fukuyama and T. Ando (Springer, Berlin, 1992). 


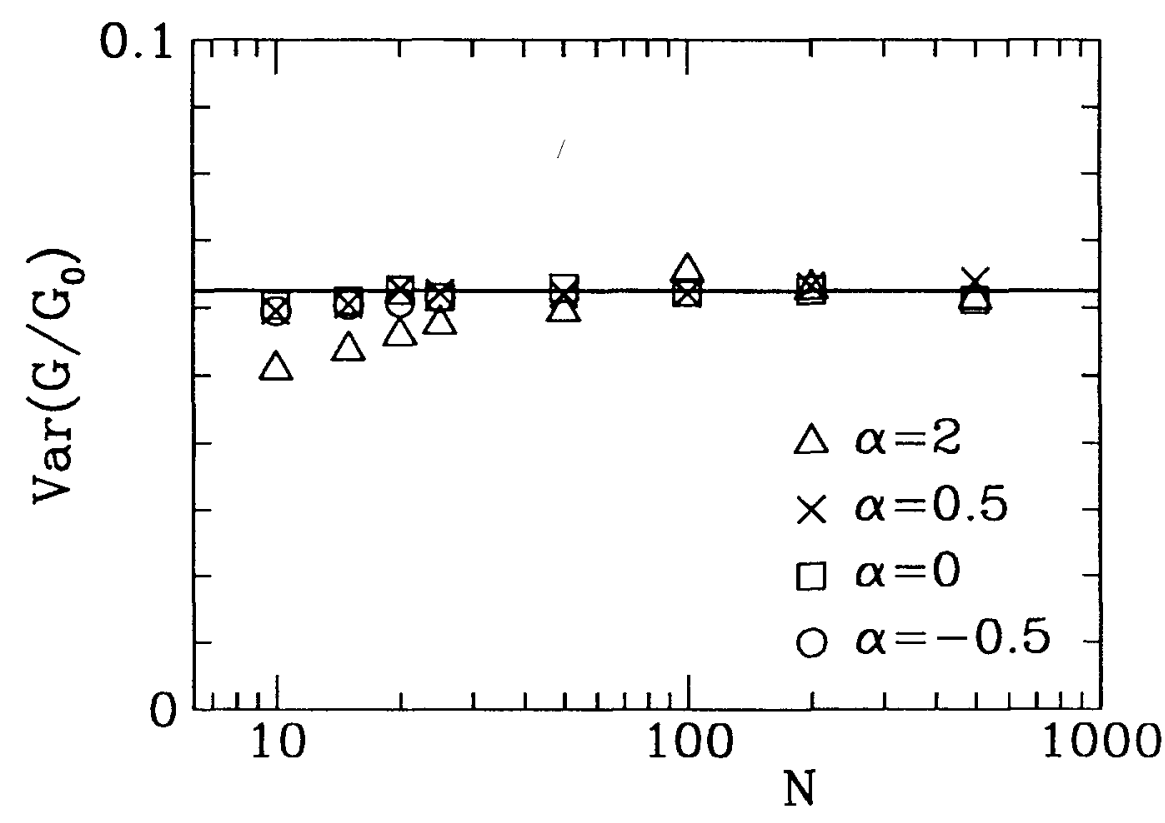

Fig. 1. Variance of the conductance $G$ (in units of $G_{0}=2 e^{2} / h$ ), as a function of the number of channels $N$. The data points are obtained by integration of the exact correlation function for the generalized Laguerre ensemble [6, 17], for various value's of the microscopic parameter $\alpha$. The estimated error in the numerical integration is \pm 0.001 . (For $\alpha=-0.5$ we could only integrate with the required accuracy for $N$ up to 25.) The horizontal line at 0.0625 is the $\alpha$-independent value predicted in the limit $N \rightarrow \infty$ by the variance formula (26).

$$
\tilde{a}(k)=\int_{-\infty}^{\infty} d x \mathrm{e}^{\mathrm{i} k x} \frac{1}{1+\mathrm{e}^{x}}=-\frac{\mathrm{i} \pi}{\sinh (\pi k)} .
$$

Substitution into Eq. (26) yields the variance

$$
\operatorname{Var}\left(G / G_{0}\right)=\beta^{-1} \int_{0}^{\infty} d k \frac{2 k}{\sinh (2 \pi k)}=\frac{1}{8} \beta^{-1} .
$$

For the Laguerre ensemble (which has $\beta=2$ ) we would thus expect from our variance formula that $\operatorname{Var}\left(G / G_{0}\right)=0.0625$ for $N \gg 1$, independent of .1 and of the parameter $\alpha$ (which in this model plays the role of a "microscopic" parameter). As one can see in Fig. 1, this is indeed what we find (within numerical accuracy) from integration of the exact correlation function.

The coefficient $\frac{1}{8}$ in Eq. (30) is close to, but not precisely identical 10 the established value $\frac{2}{15}$ for a quasi-one-dimensional conductor $[2,18]$. Th" smallness of the difference explains why it was not noticed previously. l'rom a practical point of view, the difference is not really significant, but conceptually it has the important implication that the probability distribution (6) is not exact. In other words, the interaction between the $\lambda$ 's is not precisely logarithmic.

\section{Applications}

The variance formula (26) can be readily applied to other transport properties which are linear statistics. As an illustration, we briefly discuss some examples which have previously been studied by other methods $[10,11,12]$.

\subsection{SHOT NOISE}

The shot-noise power $P$ of a phase-coherent conductor is given by [19]

$$
P / P_{0}=\sum_{n=1}^{N} T_{n}\left(1-T_{n}\right)=\sum_{n=1}^{N} \frac{\lambda_{n}}{\left(1+\lambda_{n}\right)^{2}},
$$

with $P_{0}=2 e|U| G_{0}$ ( $U$ is the applied voltage). The Mellin transform of $a(\lambda)=\lambda(1+\lambda)^{-2}$ is

$$
\tilde{a}(k)=\int_{-\infty}^{\infty} d x \mathrm{e}^{\mathrm{i} k x} \frac{\mathrm{e}^{x}}{\left(1+\mathrm{e}^{x}\right)^{2}}=\frac{\pi k}{\sinh (\pi k)} .
$$

Ilence the variance becomes

$$
\operatorname{Var}\left(P / P_{0}\right)=\beta^{-1} \int_{0}^{\infty} d k \frac{2 k^{3}}{\sinh (2 \pi k)}=\frac{1}{64} \beta^{-1} .
$$

\subsection{NORMAL-SUPERCONDUCTOR INTERFACE}

The conductance $G_{\mathrm{NS}}$ of a disordered microbridge between a normal and a superconducting reservoir is related to the transmission eigenvalues in the normal state by [20]

$$
G_{\mathrm{NS}} / G_{0}=2 \sum_{n=1}^{N}\left(\frac{T_{n}}{2-T_{n}}\right)^{2}=\sum_{n=1}^{N} \frac{2}{\left(1+2 \lambda_{n}\right)^{2}} .
$$

This expression holds only in zero magnetic field and for spin-independent scattering, i.e. for $\beta=1$. The Mellin transform of $a(\lambda)=2(1+2 \lambda)^{-2}$ is

$$
\tilde{a}(k)=\int_{-\infty}^{\infty} d x \mathrm{e}^{\mathrm{i} k x} \frac{2}{\left(1+2 \mathrm{e}^{x}\right)^{2}}=-\frac{2 \pi k+2 \pi \mathrm{i}}{\sinh (\pi k)} \mathrm{e}^{-\mathrm{i} k \ln 2},
$$

which leads to the variance

$$
\operatorname{Var}\left(G_{\mathrm{NS}} / G_{0}\right)=8 \int_{0}^{\infty} d k \frac{k^{3}+k}{\sinh (2 \pi k)}=\frac{9}{16},
$$

where we have set $\beta$ equal to 1 . 